\title{
Fluids confined in wedges and by edges: From cluster integrals to thermodynamic properties referred to different regions
}

\author{
Ignacio Urrutia ${ }^{\dagger *}$ \\ ${ }^{\dagger}$ Departamento de Física de la Materia Condensada, Centro Atómico Constituyentes, \\ CNEA, Av.Gral. Paz 1499, 1650 Pcia. de Buenos Aires, Argentina and
}

CONICET

\begin{abstract}
Recently, new insights in the relation between the geometry of the vessel that confines a fluid and its thermodynamic properties were traced through the study of cluster integrals for inhomogeneous fluids. In this work I analyze the thermodynamic properties of fluids confined in wedges or by edges, emphasizing on the question of the region to which these properties refer. In this context, the relations between the line-thermodynamic properties referred to different regions are derived as analytic functions of the dihedral angle $\alpha$, for $0<\alpha<2 \pi$, which enables a unified approach to both edges and wedges. As a simple application of these results, I analyze the properties of the confined gas in the low-density regime. Finally, using recent analytic results for the second cluster integral of the confined hard sphere fluid, the low density behavior of the line thermodynamic properties is analytically studied up to order two in the density for $0<\alpha<2 \pi$ and by adopting different reference regions.
\end{abstract}

\section{INTRODUCTION}

The interest on confined inhomogeneous fluids covers a large length of scales of the particles size which starts at the simplest one-atom per molecule (e.g. the noble gases) and goes up to proteins, polymers (including DNA molecules), and large colloids.[1-5] The thermodynamic properties of these systems are influenced by the geometry of the vessel or substrate that constrains the spatial region where the molecules of the system are enabled to move. Important efforts are continuously devoted to reach a detailed description of the response of fluids to some simple geometrical constraints like the confinement in pores with slit, cylindrical and spherical shapes, as well as the case of fluids in contact with planar and curved walls.

This work focuses on fluids confined by open dihedrons built by two planar faces that meet in an edge. Previous studies were dedicated to analyze the adsorption of liquid-vapor coexisting phases on edges and wedges, [6-9] and also, to the adsorption on corrugated surfaces.[1012] The main characteristic of the thermodynamics of fluids confined by edges and wedges is the existence of line tensions. This property also characterizes systems with two coexisting phases adsorbed on planar substrates (sessile drops) and systems with three coexisting phases that meet on a common line. $[13,14]$

One of the particularities of the edge/wedge type of confinement is that it produces non-trivial spatial inhomogeneities of the fluid. As in the case of fluids confined by curved walls, it happens that different points of view in the very beginning of the analysis produce dissimilar properties.[15] Thus, it is relevant to establish the basis that allow us to compare the thermodynamic properties found by adopting these different points of view.

\footnotetext{
* iurrutia@cnea.gov.ar
}

In this work, I analyze the statistical mechanics and thermodynamic properties of a fluid confined in an edge/wedge on the basis of the representation of its grand potential in powers of the activity. In Sec. II different type of edge/wedge confinements are discussed and the thermodynamics of the fluid composed by spherical particles is revisited. There, I analyze the free energy and related thermodynamic magnitudes of the confined fluid emphasizing on the explicit choice of the reference region to which system properties refer. Sec. III describes the functional dependence of the cluster integrals with the measures of the edge/wedge spatial region and the consequences that follow on system properties. There, new relations between bulk- surface- and line-thermodynamic properties for different reference regions, are shown. They take the form of transformation laws and apply to any density. Also, the behavior of low density gases is discussed. In Sec. IV, this approach is utilized to derive analytic expressions for the thermodynamic properties (pressure, surface tension, line tension, surface- and linear- adsorptions) of the confined hard sphere fluid up to order two in density. The consequences of adopting different reference regions on the thermodynamics of this system are also discussed in this section. The expressions obtained of line-tension and line-adsorption, show the dependence with the opening dihedral angle. Final remarks are presented in Sec. V.

\section{DETAILED DESCRIPTION OF A FLUID IN AN EDGE/WEDGE CONFINEMENT}

Let us consider an open system of particles at constant temperature $T$ and chemical potential $\mu$, which is confined by two planar walls that intersect in an edge. For simplicity we only refer here to spherical particles. The walls exert a hard potential $\phi(\mathbf{r})$ that constrains the position of the center of each particle to a region $\mathcal{A}$ with dihedral shape (throughout this work the open-dihedron 


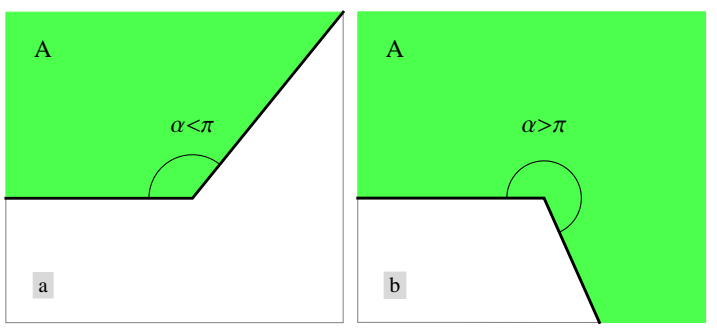

Figure 1. Fluid confined by a hard-wall dihedron. In the region $\mathcal{A}$ in light-gray (green) particles are free to move while the region in white is forbidden. Note that no matter the value of the opening angle both light-gray (green) and white regions are straight-edge dihedrons.
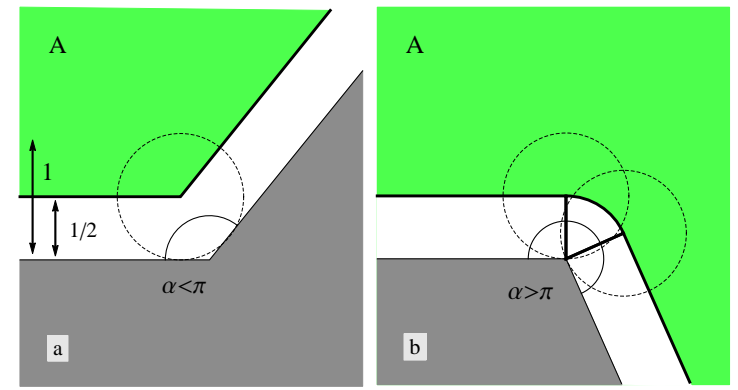

Figure 2. Fluid confined by a hard wall dihedron that induce an excluded region. Painted with darker gray is the hard wall, lighter gray (green) corresponds to the fluid and the excluded region is in white. (a) shows the case $\alpha<\pi$ while (b) corresponds to the case $\alpha>\pi$. The wall-particle hard repulsion distance is $\sigma / 2$ and dashed circles represent particles at selected positions near the edge/wedge. The arrows show characteristic lengths in $\sigma$ units.

geometrical shape is referred to as dihedron) being $\alpha$ the inner angle between faces (inner to $\mathcal{A}$ ). I analyze two different types of edge/wedge confinement. Fig.1 shows one of the edge/wedge confinement considered. There, the edge/wedge available region $\mathcal{A}$ is defined by the Boltzmann factor $\exp [-\phi(\mathbf{r}) / k T]=\Theta(|\mathbf{r}-\mathcal{C}|)$ where $k$ is the Boltzmann's constant, $\Theta(x)$ the Heaviside function $[\Theta(x)=1$ if $x>0$ and zero otherwise], the dihedral region which is the complement of $\mathcal{A}$ is $\mathcal{C}=\mathbb{R}^{3} \backslash \mathcal{A}$, and $|\mathbf{r}-\mathcal{C}|$ is the shortest distance between $\mathbf{r}$ and $\mathcal{C}$. Note that the faces of $\mathcal{A}$ meet on a straight line, thus, I call it a straight-edge dihedron. On the other hand, one has the confinement defined by the Boltzmann factor $\exp [-\phi(\mathbf{r}) / k T]=\Theta\left(|\mathbf{r}-\mathcal{C}|-\frac{\sigma}{2}\right)$ being $\mathcal{C}$ a solid dihedral region and $\sigma / 2$ the minimum distance between the center of a particle and the solid dihedron. The latter case, particularly relevant for colloidal particles and macromolecules, is drawn in Fig. 2 where the forbidden region between $\mathcal{A}$ and $\mathcal{C}$ is also indicated. Note that for $0<\alpha<\pi$ the region $\mathcal{A}$ is a straight-edge dihedron (shown in Fig. 2a). On the contrary, for $\pi<\alpha<2 \pi$ (shown in Fig. 2b) $\mathcal{A}$ is a rounded-edge dihedron (it has a curved end-of-fluid surface). This kind of rounded edge confinement is produced by the external hard potential being the inter-particles potential arbitrary. In summary, Figs. 1a, 1b and 2a correspond to a straight-edge confinement while Fig. 2b corresponds to a rounded-edge.

Before analysing the thermodynamic properties of this confined fluid it is necessary to adopt a region $\mathcal{B}$ as the reference region (RR). $[15,16]$ Note that $\mathcal{B}$ may coincide or not with $\mathcal{A}$. I wish to underline that in the study of confined fluids is crucial to clearly establish the adopted $\mathrm{RR}$ which fix the position and shape of its boundary the surface of tension. This question is as important as to establish the system of reference in the study of a mechanical system. Hence, I adopt as RR the region $\mathcal{B}$ which nearly follows the shape of $\mathcal{A}$ and has measures $\mathbf{M}_{\mathcal{B}}=(V, A, L)$ (being $V, A$ and $L$ the volume, surface area and length of the edge of $\mathcal{B}$, respectively). A detailed analysis of different prescriptions for $\mathcal{B}$ is presented in Sec.III. The grand potential of the confined fluid, relative to $\mathcal{B}$, can be written as

$$
\Omega=-P V+\gamma A+\mathcal{T} L
$$

where $P$ is the pressure of the fluid, $\gamma$ is the wall/fluid surface tension (or surface free-energy), and $\mathcal{T}$ the wall/fluid line tension (or line free-energy). The mean number of particles in the confined system is

$$
\begin{aligned}
N & =-\frac{\partial \Omega}{\partial \mu}, \\
& =\rho V+\Gamma_{A} A+\Gamma_{L} L,
\end{aligned}
$$

where $\rho$ is the mean number density, $\Gamma_{A}$ is the excess adsorption per unit area, and $\Gamma_{L}$ is the excess adsorption per unit length. Naturally, this kind of linear decomposition also applies to other magnitudes, such as the entropy $S=-\frac{\partial \Omega}{\partial T}$, the energy $U=\Omega+T S+\mu N$, and higher order derivatives like $\sigma_{N}^{2} \equiv\left\langle N^{2}\right\rangle-N^{2}=k T \frac{\partial N}{\partial \mu}$ which describe fluctuations.

From Eq. (1) is clear that once $\mathcal{A}$ is fixed, $\Omega$ becomes independent of the adopted RR. On the contrary, since measures are relative to $\mathcal{B}$, some of the magnitudes $(P, \gamma, \mathcal{T})$ depend on $\mathcal{B}$. Naturally, the same argument shows that $N$ is independent of the adopted RR although some of the magnitudes $\left(\rho, \Gamma_{A}, \Gamma_{L}\right)$ may depend on $\mathcal{B}$, and so on. In summary, even when we have an idea of the meaning of the magnitudes $\left(P, \gamma, \mathcal{T}, \rho, \Gamma_{A}, \Gamma_{L}\right)$ that allow us to give a name to each one, they were not appropriately defined yet. Indeed, they do not describe the pure properties of the confined fluid (as it may be suggested by the adopted names for these magnitudes), but they describe the properties of the fluid with regard to a given RR in a sense that will be clarified below. Some general aspects of the former discussion follow the analysis of macroscopic systems with coexisting phases done in Ref. [14]. 


\section{CLUSTER INTEGRALS AND THERMODYNAMICS}

Let us consider a system of particles interacting through a pair potential $\psi(r)$ with finite range. The center of these particles is confined by a hard external potential to an edge/wedge region $\mathcal{A}$. It was recently shown that the cluster integrals of the system take the form $[17,18]$

$$
\tau_{i}=i ! b_{i} V-i ! a_{i} A+i ! c_{i} L .
$$

Here, the $i$-th cluster integral $\tau_{i}$ is linear on the extensive measures $\mathbf{M}_{\mathcal{A}}=(V, A, L)$ that geometrically characterize the region $\mathcal{A}$ (its volume $V$, surface area $A$, and the length of its edge $L$ ). We say that $\mathbf{M}_{\mathcal{A}}$ are the measures of the system relative to $\mathcal{A}$. Besides, cluster integral also depends on the opening dihedral angle between faces, $\alpha$. The volume coefficients $b_{i}$ are the well known Mayer's cluster integrals for homogeneous systems and the area coefficients $a_{i}$ were introduced to describe a fluid adsorbed on an infinite wall.[19,20] $b_{i}$ and $a_{i}$ with $i>1$ depend on $\psi(r)$ but are independent of $\alpha$, being $\tau_{1}=b_{1} V$ with $b_{1}=1$ and $V=Z_{1}$, the configuration integral of one particle. Eq. (4) was originally derived for the case of a straight-edge dihedral region[17] $\mathcal{A}$, i.e. the cases described in Figs. 1a, 1b and 2a, and was latter extended to the rounded-edge dihedron shown in Fig.2b.[16]

It is well known that in the low density regime or gaseous phase the properties of the confined fluid can be rigorously written as power series in the activity $z=\Lambda^{-3} \exp (\beta \mu)$ (here $\beta=1 / k T$ is the inverse temperature and $\Lambda$ the de Broglie's thermal length).[18] The Mayer series of the grand potential for the confined fluid is given by

$$
\Omega=-\beta^{-1} \sum_{i \geq 1} \frac{\tau_{i}}{i !} z^{i}
$$

being its mean number of particles

$$
N=\sum_{i \geq 1} i \frac{\tau_{i}}{i !} z^{i}
$$

By replacing Eq. (4) in Eqs. (5) and (6), one obtains

$$
\begin{aligned}
& \beta \Omega=-\left(\sum_{i \geq 1} b_{i} z^{i}\right) V+\left(\sum_{i \geq 1} a_{i} z^{i}\right) A-\left(\sum_{i \geq 1} c_{i} z^{i}\right) L, \\
& N=-\left(\sum_{i \geq 1} i b_{i} z^{i}\right) V-\left(\sum_{i \geq 1} i a_{i} z^{i}\right) A+\left(\sum_{i \geq 1} i c_{i} z^{i}\right) L .
\end{aligned}
$$

Consistently, a similar transformation applies to other thermodynamic magnitudes $\left[S, U, \sigma_{N}^{2}\right.$, see Eq. (3)]. Eqs. (1) and (7) have a similar dependence with the measures. Nevertheless, Eq. (1) is in terms of measures $\mathbf{M}_{\mathcal{B}}$ that correspond to the choice $\mathcal{B}$ as RR, while Eq. (7) is in terms of measures regarding to $\mathcal{A}$ as RR. Of course, the same applies to Eqs. (3) and (8). Eqs. (1-3) show the advantages in deriving $\tau_{i}$ as a linear function of the measures of $\mathcal{B}$. If it is possible, one find the $z$ power series for the intensive thermodynamic properties $P, \gamma, \mathcal{T}$ and densities $\rho, \Gamma_{A}, \Gamma_{L}$, referred to $\mathcal{B}$.

Eq. (4) transpires lineal algebra concepts. It can be seen as a vector in an abstract space with basis of coordinates $(V, A, L)$ and components $\left(i ! b_{i},-i ! a_{i}, i ! c_{i}\right)$ but also as the inner product $\left(i ! b_{i},-i ! a_{i}, i ! c_{i}\right) \cdot \mathbf{M}_{\mathcal{A}}$ between row and column vectors that live in dual spaces. These analogies flow to Eqs. $(7,8)$ and will be further investigated in the following Secs. III A and IIIB for different ranges of $\alpha$.

The confinement of the systems drawn in Fig. 1 is purely characterized by the region $\mathcal{A}$ where the density distribution is non-null. This density-based choice of $\mathcal{B}$ will be labeled with a d subindex (d-RR). For the confinement shown in Figs. $1 \mathrm{a}$ and $1 \mathrm{~b}$ the unique simple choice for RR is $\mathcal{A}$ itself, this prevents to analyze them from the point of view of the freedom to choose the RR.

On the other hand, even when the systems shown in Fig. 2 can also be analyzed under the same densitybased $\mathcal{B}$, other RR could be adopted. To analyze this problem and the relation between the thermodynamic properties obtained under different choices of $\mathcal{B}$, we study the edges/wedges with angles $0<\alpha<\pi$ and $\pi<\alpha<2 \pi$ separately. It is interesting to note that when the d-RR is adopted the system depicted in Fig. 1a and the system drawn in Fig. 2a are identical, and thus, their properties are identical too.

\section{A. Case I $(0<\alpha<\pi)$}

For the case of the wedge confinement drawn in Fig. 2a, I study two different choices for the RR that are the most natural to be adopted. Under the density-based $\mathrm{RR}$ that identifies $\mathcal{B}$ with $\mathcal{A}$, the measures are $\mathbf{M}_{\mathrm{d}}=$ $\left(V_{\mathrm{d}}, A_{\mathrm{d}}, L\right)$, being the $i$-th cluster integral

$$
\tau_{i} / i !=b_{i} V_{\mathrm{d}}-a_{i} A_{\mathrm{d}}+c_{i}(\beta) L=\mathbf{b}_{\mathrm{d}} \cdot \mathbf{M}_{\mathrm{d}},
$$

where $\mathbf{b}_{\mathrm{d}}=\left(b_{i},-a_{i}, c_{i}\right)$ is the vector of coefficients. The second simple choice for $\mathcal{B}$ is the empty-region (e-RR), i.e. $\mathcal{B}$ is taken as $\mathcal{A}$ joined with the white region in Fig. 2a and the measures are $\mathbf{M}_{\mathrm{e}}=\left(V_{\mathrm{e}}, A_{\mathrm{e}}, L\right)$. In what follows we will use $\sigma$ (see Fig. 2) as the unit length. From geometrical considerations it is possible to obtain the linear relation between both sets of measures: $V_{\mathrm{d}}=V_{\mathrm{e}}-\frac{1}{2} A_{\mathrm{e}}+\frac{1}{4} \cot \frac{\alpha}{2} L$ for $0<\alpha<\pi, A_{\mathrm{d}}=A_{\mathrm{e}}-\csc \alpha L$ for $0<\alpha<\frac{\pi}{2}$, and $A_{\mathrm{d}}=A_{\mathrm{e}}-\cot \frac{\alpha}{2} L$ for $\frac{\pi}{2}<\alpha<\pi$. Thus, under the e-RR choice

$$
\tau_{i} / i !=\tilde{b}_{i} V_{\mathrm{e}}-\tilde{a}_{i} A_{\mathrm{e}}+\tilde{c}_{i}(\alpha) L=\mathbf{b}_{\mathrm{e}} \cdot \mathbf{M}_{\mathrm{e}} .
$$

We introduce the matrix $Y$ that transforms between both sets of measures

$$
\mathbf{M}_{\mathrm{d}}=Y \cdot \mathbf{M}_{\mathrm{e}} \text { and } \mathbf{M}_{\mathrm{e}}=Y^{-1} \cdot \mathbf{M}_{\mathrm{d}} .
$$


Its expression follows from the relations above Eq. (10)

$$
Y=\left(\begin{array}{ccc}
1 & -\frac{1}{2} & \frac{1}{4} \cot \frac{\alpha}{2} \\
0 & 1 & -y(\alpha) \\
0 & 0 & 1
\end{array}\right)
$$

with $y(\alpha)=\csc \alpha$ if $0<\alpha<\frac{\pi}{2}$ and $y(\alpha)=\cot \frac{\alpha}{2}$ if $\frac{\pi}{2}<\alpha<\pi$ (note that $y(\alpha)$ is a continuous non-derivable function at $\left.\alpha=\frac{\pi}{2}\right)$. Given that $\Omega$ remains unmodified no matter which RR is adopted, one finds the linear relation between the unknown coefficients $\mathbf{b}_{\mathrm{e}}=\left(\tilde{b}_{i},-\tilde{a}_{i}, \tilde{c}_{i}\right)$ and the known $\mathbf{b}_{\mathrm{d}}$, through the $Y$ matrix

$$
\begin{aligned}
\mathbf{b}_{\mathrm{e}} \cdot \mathbf{M}_{\mathrm{e}} & =\mathbf{b}_{\mathrm{d}} \cdot Y \cdot Y^{-1} \cdot \mathbf{M}_{\mathrm{d}}, \\
\mathbf{b}_{\mathrm{e}} & =\mathbf{b}_{\mathrm{d}} \cdot Y .
\end{aligned}
$$

Besides, through Eqs. (1-3) $Y$ also transforms the thermodynamic properties

$$
\begin{aligned}
& (-P, \gamma, \mathcal{T})_{\mathrm{e}}=(-P, \gamma, \mathcal{T})_{\mathrm{d}} \cdot Y, \\
& \left(\rho, \Gamma_{A}, \Gamma_{L}\right)_{\mathrm{e}}=\left(\rho, \Gamma_{A}, \Gamma_{L}\right)_{\mathrm{d}} \cdot Y,
\end{aligned}
$$

where the label outside brackets shows the adopted reference region. These relations should be valid even when the series expansion in powers of $z$ does not apply, and thus, they should apply to any density. Eqs. (14, 15), with $Y$ taken from Eq. (12), are one of the main results of the current work. They show the transformation law between the intensive properties of the confined system when different RRs are adopted.

Now, we turn our attention to Eqs. $(1,7)$ and (14). They show that $P_{\mathrm{e}}=P_{\mathrm{d}}=P$ being $P$ the pressure of the bulk fluid at the same $T$ and $\mu$. Furthermore, one obtains

$$
\gamma_{\mathrm{e}}=\gamma_{\mathrm{d}}+P / 2
$$

Eq. (16) found here for a wedge confinement is a known relation for fluids adsorbed on both planar and curved walls. $[15,20]$ The $z$ power series representation of $\gamma_{\mathrm{e}}$ and $\gamma_{\mathrm{d}}$ shows that they are the surface tension of the fluid in contact with an infinite planar wall (each one for a different RR). The line tension transforms as

$$
\mathcal{T}_{\mathrm{e}}=\mathcal{T}_{\mathrm{d}}-\gamma_{\mathrm{d}} y(\alpha)-\frac{P}{4} \cot \frac{\alpha}{2}
$$

To the best of my knowledge it is the first time that Eq. (17), which applies to any density, is derived. Turning to Eqs. $(3,8)$ and $(15)$, they imply that $\rho_{\mathrm{e}}=\rho_{\mathrm{d}}=\rho$ with $\rho$ the number density of the bulk fluid (at the same $T$ and $\mu)$. For the surface adsorption one finds,

$$
\left(\Gamma_{A}\right)_{\mathrm{e}}=\left(\Gamma_{A}\right)_{\mathrm{d}}-\rho / 2,
$$

which is known to be an exact relation for planar walls. Again, based on the $z$ power series one finds that both $\left(\Gamma_{A}\right)_{\mathrm{e}}$ and $\left(\Gamma_{A}\right)_{\mathrm{d}}$ are the adsorption of the fluid on an infinite planar wall (each one for a different RR). Furthermore, one obtains for the excess linear adsorption

$$
\left(\Gamma_{L}\right)_{\mathrm{e}}=\left(\Gamma_{L}\right)_{\mathrm{d}}-\left(\Gamma_{A}\right)_{\mathrm{d}} y(\alpha)+\frac{\rho}{4} \cot \frac{\alpha}{2} .
$$

Again, this expression applies to any density and it was never published before.

\section{B. Case II $(\pi<\alpha<2 \pi)$}

Now, focusing on the case shown in Fig. 2b, I will analyze two different choices for the region $\mathcal{B}$, which split in three different sets of measures that are the most natural to adopt. The first choice is the d-RR, which corresponds to identify $\mathcal{B}$ with $\mathcal{A}$. For this d-RR one can consider two different criteria to define the measures depending on whether $A$ is taken as the area of the planar part $\left(A_{p \mathrm{~d}}\right)$ of the surface $\partial \mathcal{B}$ or as its total area. Thus, using the first criteria the measures are $\mathbf{M}_{\mathrm{d} 1}=\left(V_{\mathrm{d}}, A_{p \mathrm{~d}}, L\right)$ and

$$
\tau_{i} / i !=b_{i} V_{\mathrm{d}}-a_{i} A_{p \mathrm{~d}}+c_{i}(\alpha) L=\mathbf{b}_{\mathrm{d} 1} \cdot \mathbf{M}_{\mathrm{d} 1},
$$

with the vector of coefficients $\mathbf{b}_{\mathrm{d} 1}=\left(b_{i},-a_{i}, c_{i}\right)$. However, if one adopts the second criteria that assumes $A$ as the total area of $\partial \mathcal{B}$ it is obtained

$$
\tau_{i} / i !=\bar{b}_{i} V_{\mathrm{d}}-\bar{a}_{i} A_{\mathrm{d}}+\bar{c}_{i}(\alpha) L=\mathbf{b}_{\mathrm{d} 2} \cdot \mathbf{M}_{\mathrm{d} 2}
$$

with $\mathbf{M}_{\mathrm{d} 2}=\left(V_{\mathrm{d}}, A_{\mathrm{d}}, L\right)$ and $A_{p \mathrm{~d}}=A_{\mathrm{d}}-(\alpha-\pi) \frac{1}{2} L$. The relationship between both sets of measures is

$$
\mathbf{M}_{\mathrm{d} 1}=Y \cdot \mathbf{M}_{\mathrm{d} 2}, \mathbf{M}_{\mathrm{d} 2}=Y^{-1} \cdot \mathbf{M}_{\mathrm{d} 1}
$$

while the vectors of coefficients relate through

$$
\mathbf{b}_{\mathrm{d} 2}=\mathbf{b}_{\mathrm{d} 1} \cdot Y
$$

with

$$
Y=\left(\begin{array}{ccc}
1 & 0 & 0 \\
0 & 1 & -\frac{1}{2}(\alpha-\pi) \\
0 & 0 & 1
\end{array}\right)
$$

The relations between the equations of state $(-P, \gamma, \mathcal{T})$ and also $\left(\rho, \Gamma_{A}, \Gamma_{L}\right)$, in $\mathrm{d} 1-\mathrm{RR}$ and $\mathrm{d} 2-\mathrm{RR}$ are given by Eqs. (14) and (15) with the obvious change of labels and with $Y$ taken from Eq. (24). Therefore, one finds $P_{\mathrm{d} 2}=P_{\mathrm{d} 1}=P$ (with $P$ the bulk pressure), $\gamma_{\mathrm{d} 2}=\gamma_{\mathrm{d} 1}$ (which are equal to the planar-wall surface tension $\gamma_{\mathrm{d}}$ discussed for the case $\alpha<\pi$ ) and

$$
\mathcal{T}_{\mathrm{d} 2}=\mathcal{T}_{\mathrm{d} 1}-\gamma_{\mathrm{d}}(\alpha-\pi) / 2
$$

Besides, it is obtained $\rho_{\mathrm{d} 2}=\rho_{\mathrm{d} 1}=\rho,\left(\Gamma_{A}\right)_{\mathrm{d} 2}=\left(\Gamma_{A}\right)_{\mathrm{d} 1}$ (which are equal to the planar-wall adsorption $\left(\Gamma_{A}\right)_{\mathrm{d}}$ discussed for the case $\alpha<\pi$ ) and

$$
\left(\Gamma_{L}\right)_{\mathrm{d} 2}=\left(\Gamma_{L}\right)_{\mathrm{d} 1}-\left(\Gamma_{A}\right)_{\mathrm{d} 1}(\alpha-\pi) / 2
$$


It seems that Eqs. (25) and (26), that apply to any density, are novel results.

The other choice for $\mathcal{B}$ is the e-RR, that is, the join of region $\mathcal{A}$ and the white region in Fig. 2b. In this case, the measures are $\mathbf{M}_{\mathrm{e}}=\left(V_{\mathrm{e}}, A_{\mathrm{e}}, L\right)$ and

$$
\tau_{i} / i !=\tilde{b}_{i} V_{\mathrm{e}}-\tilde{a}_{i} A_{\mathrm{e}}+\tilde{c}_{i}(\alpha) L=\mathbf{b}_{\mathrm{e}} \cdot \mathbf{M}_{\mathrm{e}},
$$

with $V_{\mathrm{d}}=V_{\mathrm{e}}-\frac{1}{2} A_{\mathrm{e}}-\frac{1}{8}(\alpha-\pi) L$ and $A_{p \mathrm{~d}}=A_{\mathrm{e}}$. Eqs. $(22,23)$ describe the transformation between both, the measures and the coefficients, they remain valid with the change of labels $\mathrm{d} 2 \rightarrow \mathrm{e}$ and for

$$
Y=\left(\begin{array}{ccc}
1 & -\frac{1}{2} & -\frac{1}{8}(\alpha-\pi) \\
0 & 1 & 0 \\
0 & 0 & 1
\end{array}\right)
$$

Yet non-surprising, following Eqs. (14) and (15) with the obvious change of labels and taking $Y$ from Eq. (28) one obtains $P_{\mathrm{e}}=P$ and $\rho_{\mathrm{e}}=\rho$. Furthermore, both $\gamma_{\mathrm{e}}$ and $\left(\Gamma_{A}\right)_{\mathrm{e}}$ coincide with the planar-wall magnitudes found for the case $\alpha<\pi$, and then Eqs. $(16,18)$ apply for the broad range $0<\alpha<2 \pi$. Finally, one obtains

$$
\begin{gathered}
\mathcal{T}_{\mathrm{e}}=\mathcal{T}_{\mathrm{d} 1}+P(\alpha-\pi) / 8, \\
\left(\Gamma_{L}\right)_{\mathrm{e}}=\left(\Gamma_{L}\right)_{\mathrm{d} 1}-\rho(\alpha-\pi) / 8 .
\end{gathered}
$$

Eqs. (29) and (30) were not published earlier.

\section{Low density}

In this brief digression the confined ideal gas and the low density regime of the confined non-ideal gas are analyzed. I first concentrate in the d-RR (d-RR for $0<\alpha<\pi$, d1- and d2-RR for $\pi<\alpha<2 \pi$ ). To obtain the properties of the confined ideal gas one truncates all the series in Eqs. $(6,7,8)$ at the first order in power of $z$. By adopting d-RR the volume $V_{\mathrm{d}}$ is equal to $Z_{1}$ and the first cluster integral is $\tau_{1}=V_{\mathrm{d}}$. Thus, $\beta P=z, \rho=z$,

$$
\gamma=\mathcal{T}=0, \text { and } \Gamma_{A}=\Gamma_{L}=0 .
$$

Therefore, under d-RR the confined ideal gas is thoroughly described by $\beta P=\rho$, i.e. the equation of state of the bulk ideal gas. Clearly, if we turn to e-RR the edge/wedge confined ideal gas has non-null surface- and line- free energies. They can be evaluated using Eq. (31) and the transformations discussed in Secs. III B and III A. The conclusion is that in order to obtain the simpler expressions for the thermodynamics of the confined ideal gas, the d-RR is better than e-RR.

For the confined non-ideal gas under d-RR the first cluster integral remains unmodified in comparison with the ideal gas. The second and higher order $\tau_{i}$ could be calculated by direct integration. Now, the series given in Eqs. $(5,6,7,8)$ are truncated at order two in $z$, which gives surface and linear thermodynamic properties proportional to $z^{2}$. Using Eqs. $(2,4)$ and trivial series manipulation one obtains the power series for $z(\rho)$ and the series representation of the thermodynamic properties in powers of $\rho$. Up to order $\rho^{2}$ it is obtained: $\beta P=\rho-b_{2} \rho^{2}$ (i.e. the virial series for the bulk gas [18]) and

$$
\beta \gamma=-\Gamma_{A} / 2, \quad \beta \mathcal{T}=-\Gamma_{L} / 2
$$

[with $\Gamma_{A}=-2 a_{2} \rho^{2}$ and $\Gamma_{L}=2 c_{2} \rho^{2}$ ]. These notable relations are not well known. They deal with inhomogeneous fluids and link linearly an excess free energy (times $\beta$ ) with the corresponding excess adsorption. It is remarkable that Eq. (32) does not include coefficients related to the interparticle potential. Eq. (32) resembles the equation of state of the bulk ideal gas, nevertheless, it applies to any edge/wedge confined fluid up to order $\rho^{2}$. As can be easily verified, the use of e-RR provides more complex expressions for the surface and linear thermodynamic properties than Eq. (32). In summary, d-RR is appropriate to obtain a simple description for the thermodynamics of the confined ideal gas and also of any gas at low density, but e-RR is not.

\section{APPLICATION TO HARD SPHERES}

Recently, through adopting the d-RR, the low density behavior of the hard sphere (HS) confined fluid in an edge/wedge cavity was studied using an analytic expression of $c_{2}(\alpha)$.[16] In this section we compare those properties with that found by adopting the e-RR. With this purpose the natural units for the HS system will be used (which is equivalent to set the particles diameter $\sigma$ as the unit length). In Ref. [16] was obtained the following exact expression

$$
c_{2}(\alpha)=-\frac{1}{15}[1+(\pi-\alpha) \cot \alpha]
$$

that applies for $0<\alpha<\pi$ in the $\mathrm{d}-\mathrm{RR}$, while the analytic expression for $\pi<\alpha<2 \pi$ in d1-RR is

$$
c_{2}(\alpha)=\frac{8}{45}(\alpha-\pi)+Q,
$$

with $Q=0.007125\{1-\exp [-2.74(\alpha-\pi)]\}$. Using the known parameters $b_{2}=-2 \pi / 3, a_{2}=-\pi / 8$ and Eqs. $(33,34)$ for $c_{2}(\alpha)$ one readily finds the series expansion of $\left\{P, \gamma, \mathcal{T}, \Gamma_{A}, \Gamma_{L}\right\}$ in power of $\rho$ up to order two, by adopting both d-RR and d1-RR. For $\mathcal{T}$ and $\Gamma_{L}$ it gives analytically the angular dependence with $\alpha$ (up to order two in $\rho$ ).

Now, we analyze the consequences of choosing a different $\mathrm{RR}$ on the thermodynamic properties of the confined HS system. In particular, novel analytic expressions of relevant line-thermodynamic properties for e-RR and d2$\mathrm{RR}$ will be derived. Through the use of Eqs. $(14,15)$, the matrices for RR transformation $(12,24,28)$, and the density power series of $P_{\mathrm{d}}, \gamma_{\mathrm{d}}, \mathcal{T}_{\mathrm{d}}, \mathcal{T}_{\mathrm{d} 1},\left(\Gamma_{A}\right)_{\mathrm{d}},\left(\Gamma_{L}\right)_{\mathrm{d}}$, 


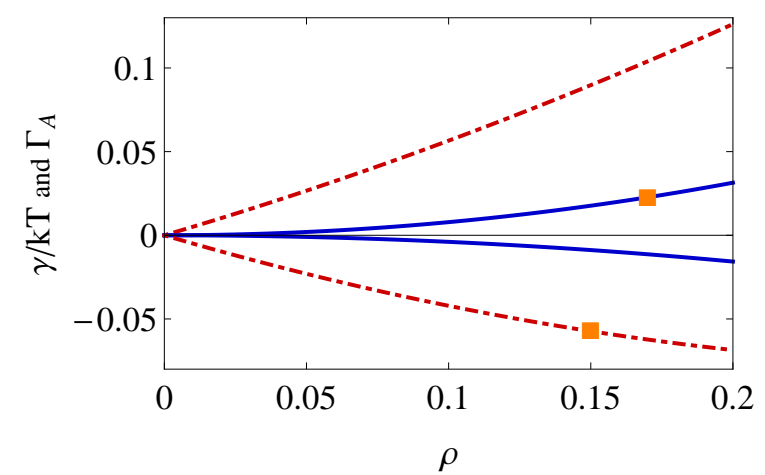

Figure 3. Surface tension and surface adsorption. Curves for adsorption are marked with squares. In continuous lines (blue) are plotted the magnitudes in d-RR while dot-dashed lines (red) refers to e-RR.

$\left(\Gamma_{L}\right)_{\mathrm{d} 1}$ one obtains the series for $\gamma, \mathcal{T}, \Gamma_{A}$ and $\Gamma_{L}$ in the e-RR and $\mathrm{d} 2-\mathrm{RR}$. For the wall-fluid surface tension and excess area-adsorption, both up to terms of order $O\left(\rho^{3}\right)$, it is obtained

$$
\begin{gathered}
\beta \gamma_{\mathrm{e}}=\frac{\rho}{2}+\frac{5 \pi}{24} \rho^{2}, \\
\left(\Gamma_{A}\right)_{\mathrm{e}}=-\frac{\rho}{2}+\frac{\pi}{4} \rho^{2} .
\end{gathered}
$$

Fig. 3 displays the surface tension and surface excess area-adsorption by adopting $\mathrm{d}-\mathrm{RR}$ and e-RR. There one can observe the effect of choosing a different $R R$ in the properties of the confined HS fluid. For the d-RR one finds $\beta \gamma_{\mathrm{d}}>0$ and $\left(\Gamma_{A}\right)_{\mathrm{d}}<0$, on the other hand for the e-RR they yield $\beta \gamma_{\mathrm{e}}<0$ and $\left(\Gamma_{A}\right)_{\mathrm{e}}>0$. Besides, near $\rho \gtrsim 0$ the null slope in $\beta \gamma_{\mathrm{d}}$ and $\left(\Gamma_{A}\right)_{\mathrm{d}}$ is apparent while $\beta \gamma_{\mathrm{e}}$ and $\left(\Gamma_{A}\right)_{\mathrm{e}}$ are linear with density. For $\mathcal{T}_{\mathrm{e}}$ in the range $0<\alpha<\pi$ there are two branches: the first one for $\alpha<\frac{\pi}{2}$ and the second one for $\alpha>\frac{\pi}{2}$, the corresponding expressions are

$$
\begin{aligned}
& \beta \mathcal{T}_{\mathrm{e}}=-\frac{\rho}{4} \cot \frac{\alpha}{2}+\frac{\rho^{2}}{15}\left[1-\left(\frac{3 \pi}{2}+\alpha\right) \cot \alpha-\frac{5 \pi}{8} \csc \alpha\right] \\
& \beta \mathcal{T}_{\mathrm{e}}=-\frac{\rho}{4} \cot \frac{\alpha}{2}+\frac{\rho^{2}}{15}\left[1+(\pi-\alpha) \cot \alpha-\frac{5 \pi}{8} \cot \frac{\alpha}{2}\right](36)
\end{aligned}
$$

In Fig. 4 it is shown the low density behavior of the edge line tension $\mathcal{T}$ for $\mathrm{d}-\mathrm{RR}$ and e-RR. In both cases, $\mathcal{T}$ is monotonous. For the $\mathbf{M}_{\mathrm{d}}$ measures $\mathcal{T}$ is positive and has positive slope. On the contrary, using $\mathbf{M}_{\mathrm{e}}$ measures $\mathcal{T}$ is negative and has a negative slope. For both, the modulus of the slope decreases with increasing $\alpha$. The obtained expression of $\left(\Gamma_{L}\right)_{\mathrm{e}}$ for $0<\alpha<\pi$ is

$$
\left(\Gamma_{L}\right)_{\mathrm{e}}=\frac{\rho}{4} \cot \frac{\alpha}{2}-\frac{2 \rho^{2}}{15}\left[1+(\pi-\alpha) \cot \alpha+\frac{15 \pi}{8} y(\alpha)\right]
$$

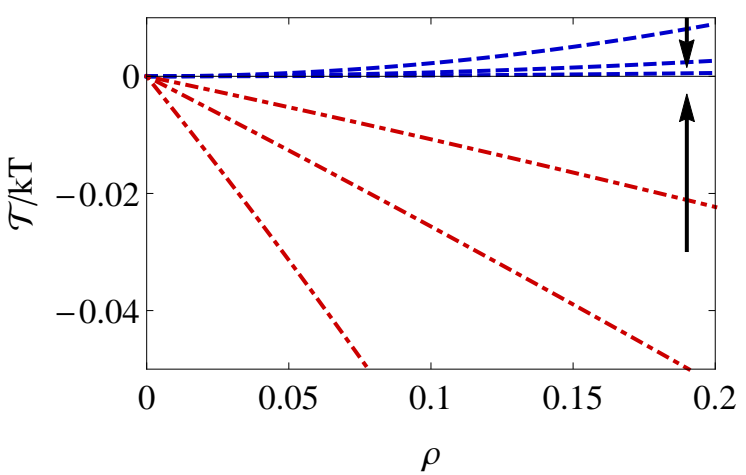

Figure 4. Line tension of the HS system vs. density for angles in the range $0<\alpha<\pi$. Continuous lines (blue) plot $\mathcal{T}_{\mathrm{d}}$ while dot-dashed lines (red) plot $\mathcal{T}_{\mathrm{e}}$. The curves correspond to $\alpha=\pi / 4, \pi / 2,3 \pi / 4$ while the arrow points to the direction of increasing values of $\alpha$.

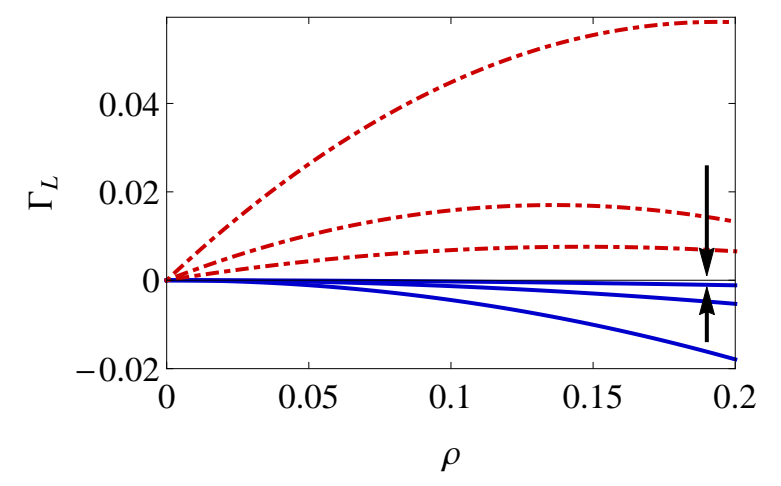

Figure 5. Line adsorption of the HS system vs. density for angles in the range $0<\alpha<\pi$, the curves correspond to $\alpha=\pi / 4, \pi / 2,3 \pi / 4$. Continuous lines (blue) plot $\left(\Gamma_{L}\right)_{\mathrm{d}}$ while dot-dashed lines (red) plot $\left(\Gamma_{L}\right)_{\mathrm{e}}$. See Fig. 4 for details.

which is non-derivable at $\alpha=\frac{\pi}{2}$ [see Eq. (12)]. Fig. 5 is similar to Fig. 4 but for the linear adsorption $\Gamma_{L}$. When the $\mathbf{M}_{\mathrm{d}}$ measures are considered the linear adsorption is monotonous, negative (i.e., there is local desorption) and has negative slope which increases with increasing $\alpha$. On the other hand, when $\mathbf{M}_{\mathrm{e}}$ measures are adopted $\Gamma_{L}$ is not monotonous, it is positive (i.e., there is local adsorption) and decreases for larger values of $\alpha$.

I also present here a similar analysis for the case $\pi<\alpha<2 \pi$. The results for the line-tension and excess linear adsorption by adopting e-RR and $\mathrm{d} 2-\mathrm{RR}$ up 


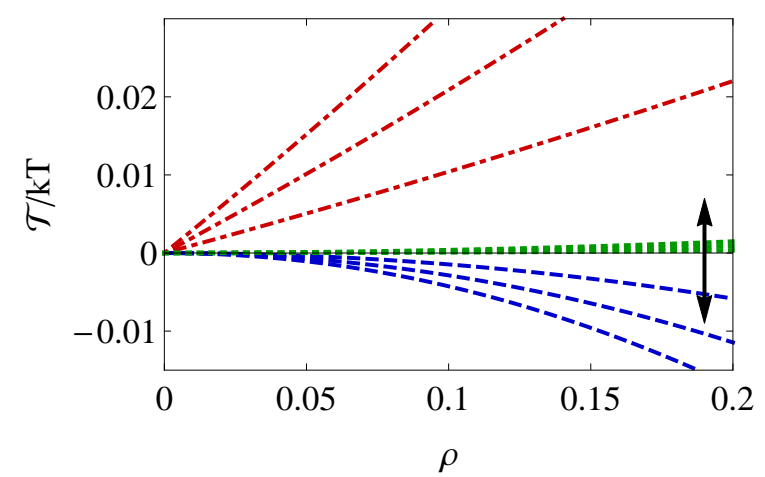

Figure 6. Line tension of the HS system vs. density for angles in the range $\pi<\alpha<2 \pi$. The curves correspond to $\alpha=5 \pi / 4,3 \pi / 2,7 \pi / 4$. In long-dashed lines (blue) it is drawn $\mathcal{T}_{\mathrm{d} 1}$, short-dashed line (green) corresponds to $\mathcal{T}_{\mathrm{d} 2}$ and dotdashed line (red) is for $\mathcal{T}_{\mathrm{e}}$. The arrows point to the direction of increasing values of $\alpha$.

to order $O\left(\rho^{3}\right)$ are

$$
\begin{gathered}
\beta \mathcal{T}_{\mathrm{e}}=\frac{\alpha-\pi}{8} \rho-\left[\left(\frac{\pi}{12}+\frac{8}{45}\right)(\alpha-\pi)+Q\right] \rho^{2} \\
\left(\Gamma_{L}\right)_{\mathrm{e}}=-\frac{\alpha-\pi}{8} \rho+\left[\frac{16}{45}(\alpha-\pi)+Q\right] \rho^{2} \\
\beta \mathcal{T}_{\mathrm{d} 2}=\left[\left(\frac{\pi}{8}-\frac{8}{45}\right)(\alpha-\pi)+Q\right] \rho^{2} \\
\left(\Gamma_{L}\right)_{\mathrm{d} 2}=\left[\left(-\frac{\pi}{4}+\frac{16}{45}\right)(\alpha-\pi)+Q\right] \rho^{2},
\end{gathered}
$$

Fig. 6 shows the linear tension of the HS system in the case $\pi<\alpha<2 \pi$. The functions $\mathcal{T}_{\mathrm{d} 1}, \mathcal{T}_{\mathrm{d} 2}$ and $\mathcal{T}_{\mathrm{e}}$ are shown for comparison. In all cases, $\mathcal{T}$ is monotonous. For the $\mathbf{M}_{\mathrm{d} 1}$ measures $\mathcal{T}$ is negative and has negative slope which decreases with increasing $\alpha$. On the other hand, for $\mathbf{M}_{\mathrm{e}}$ and $\mathbf{M}_{\mathrm{d} 2}$ measures, $\mathcal{T}$ is positive and has a positive slope which increases with increasing $\alpha$. Even, $\mathcal{T}$ for $\mathbf{M}_{\mathrm{d} 2}$ is nearly zero in the adopted scale. From the comparison between Fig. 6 and Fig. 4 the inversion of the sign of $\mathcal{T}_{\mathrm{e}}$ at $\alpha=\pi$ is evident, where the edge/wedge disappears. Fig. 7 plots the linear adsorption of the HS system for the same three measure sets. In the cases of $\mathbf{M}_{\mathrm{d} 1}$ and $\mathbf{M}_{\mathrm{d} 2}$ we observe a monotonous $\Gamma_{L}$. For the $\mathbf{M}_{\mathrm{d} 1}$ measures $\Gamma_{L}$ is a positive (i.e., there is local adsorption) increasing function and its slope increases with increasing $\alpha$. On the contrary, for $\mathbf{M}_{\mathrm{d} 2}$ measures $\Gamma_{L}$ is a negative (local desorption) decreasing function and its slope decreases with increasing $\alpha$. Even, $\Gamma_{L}$ for $\mathbf{M}_{\mathrm{d} 2}$ is nearly zero in the adopted scale. For $\mathbf{M}_{\mathrm{e}}$ measures $\Gamma_{L}$ is not monotonous, is negative (i.e., there is local desorption), attains its minimum near $\rho \simeq 0.17$ and decreases

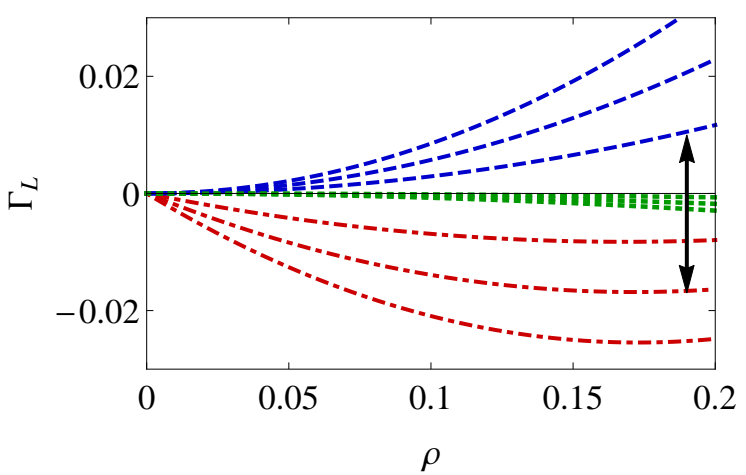

Figure 7. Line adsorption of the HS system vs. density for angles in the range $\pi<\alpha<2 \pi$. In long-dashed lines (blue) it is draw $\left(\Gamma_{L}\right)_{\mathrm{d} 1}$, short-dashed line (green) corresponds to $\left(\Gamma_{L}\right)_{\mathrm{d} 2}$ and dot-dashed line (red) is for $\left(\Gamma_{L}\right)_{\mathrm{e}}$. See Fig. 6 for more details.

with increasing values of $\alpha$. Fig. 7 and Fig. 5 show the inversion of the sign of $\left(\Gamma_{L}\right)_{\mathrm{e}}$ when both edge and wedge disappear at $\alpha=\pi$.

In the literature, both $\mathrm{d}-\mathrm{RR}$ and e-RR were used to study HS systems confined in cavities with different geometries.[21, 22] The behavior found with d-RR (including d1-RR and d2-RR) and e-RR, for both line tension and line free energy, shows that they strongly depend on the adopted reference system. Linear thermodynamic magnitudes that are less dependent on the adopted reference region can be found by considering the mean values of excess density and excess free energy in a region with finite size around the edge.[16]

\section{FINAL REMARKS}

In this work I studied the relations between the thermodynamic properties of fluids confined by wedges and edges when different RRs are adopted. The analysis was based on the activity series expansion of the grand free energy for inhomogenous systems, and on the properties of its coefficients the cluster integrals. I utilized a simple approach that linearly connects the geometric measures: volume, surface area and edge length, in the different RR that are considered. From that, the law of transformation of thermodynamic properties between RRs was deduced. A similar method was previously used to study a system of hard spheres confined by curved walls. The method was here refined and can be used to analyze inhomogenous fluids confined by walls with a variety of shapes. Under this non-standard approach I have studied the dependence of the linear-thermodynamic properties on the adopted $\mathrm{RR}$ along the complete range of 
dihedral angles $0<\alpha<2 \pi$. Analytic expressions that transform the thermodynamic intensive properties: pressure, surface tension and line tension of the system when different RR are adopted were derived for the first time. Surface adsorption and line adsorption were also analyzed in this framework. The relevant results were given in Eqs. (17, 19, 25-26, 29-30). Furthermore, the thermodynamic properties of both, the confined ideal gas and of the confined real gases at low density (up to order $\rho^{2}$ ), were analyzed by adopting different RR. We found that the density-based d-RR is advantageous to obtain a simpler analytic description of the studied properties.

Regarding to the confined HS fluid, which is a relevant reference system both for simple and colloidal fluids, the dependence of line adsorption and line tension with the edge/wedge dihedral angle and density was analyzed.
We found explicit analytic expressions truncated to order two in density that describe these properties for different $\mathrm{RR}$ and for the complete range $0<\alpha<2 \pi$. They are shown in Eqs. $(36,37,38,39)$. The new results obtained for HS complement those recently published.[16] Given that these analytic expressions are exact or quasi-exact, they constitute well defined references that should enable to validate other approximate theories like fundamental measure density functional approaches to edge/wedge confined fluids at low density.

\section{ACKNOWLEDGMENTS}

This work was supported by Argentina Grant ANPCyT PICT-2011-1887.
[1] J. R. Henderson, Physical Review E 73, 010402 (2006).

[2] L. Almenar and M. Rauscher, Journal of Physics: Condensed Matter 23, 184115 (2011).

[3] K. F. Freed and C. Wu, Journal of Chemical Physics 135, 144902 (2011).

[4] J. F. Lutsko, The Journal of Chemical Physics 137, 154903 (2012).

[5] A. Statt, A. Winkler, P. Virnau, and K. Binder, Journal of Physics: Condensed Matter 24, 464122 (2012).

[6] K. Rejmer, S. Dietrich, and M. Napiórkowski, Phys. Rev. E 60, 4027 (1999).

[7] J. R. Henderson, Physica A: Statistical Mechanics and its Applications 305, 381 (2002).

[8] J. R. Henderson, The Journal of Chemical Physics 120, 1535 (2004).

[9] J. R. Henderson, Phys. Rev. E 69, 061613 (2004).

[10] M. Schneemilch, N. Quirke, and J. R. Henderson, The Journal of Chemical Physics 118, 816 (2003).

[11] P. Bryk, R. Roth, M. Schoen, and S. Dietrich, EPL (Europhysics Letters) 63, 233 (2003).
[12] M. Schoen, Colloids and Surfaces A: Physicochemical and Engineering Aspects 206, 253 (2002).

[13] T. Getta and S. Dietrich, Phys. Rev. E 57, 655 (1998).

[14] L. Schimmele, M. Napiórkowski, and S. Dietrich, The Journal of Chemical Physics 127, 164715 (2007).

[15] I. Urrutia, Phys. Rev. E 89, 032122 (2014).

[16] I. Urrutia, The Journal of Chemical Physics 141, 244906 (2014).

[17] I. Urrutia, ArXiv e-prints (2013), arXiv:1303.3468 [condmat.stat-mech].

[18] T. L. Hill, Statistical Mechanics (Dover, New York, 1956).

[19] A. Bellemans, Physica 28, 493 (1962).

[20] J. H. Yang, A. J. Schultz, J. R. Errington, and D. A. Kofke, The Journal of Chemical Physics 138, 134706 (2013).

[21] R. Roth, Journal of Physics: Condensed Matter 22, 063102 (2010).

[22] Z. Wang and L. Liu, Phys. Rev. E 86, 031115 (2012). 